\title{
Determinação de minoxidil em formulações farmacêuticas usando permanganometria
}

\author{
Rogério Adelino de Sousa ${ }^{1}$, Éder Tadeu Gomes Cavalheiro ${ }^{2 *}$. \\ 1 - Departamento de Química, Universidade Federal de São Carlos, Rod. Washington Luiz Km \\ 235, São Carlos, São Paulo, Brasil, CEP 13565-905, Caixa Postal 676. \\ 2 - Departamento de Química e Física Molecular, Instituto de Química de São Carlos, Universidade de São Paulo, Av Trabalhador São- \\ carlense, 400, Centro, São Carlos, São Paulo, Brasil, CEP 13560-970, Caixa Postal 780. \\ *Corresponding author: Prof. Dr. Éder Tadeu Gomes Cavalheiro, Departamento de Química e Física Molecular-DQFM, Instituto de \\ Química de São Carlos / USP, Avenida do Trabalhador São-Carlense, 400, CxP 780, CEP 13560-970 - São Carlos/SP - Brasil, Telefone: \\ +551633738054 FAX: +551633739987 \\ E-mail: cavalheiro@iqsc.usp.
}

\begin{abstract}
Resumo:Um método clássico para a determinação de minoxidil em formulações farmacêuticas é proposto baseado na reação redox entre $\mathrm{KMnO}_{4}$ e o minoxidil. Os melhores resultados na permanganometria foram obtidos usando concentrações de $1,00 \times 10^{-2} \mathrm{~mol} \mathrm{~L}^{-1}$ para o permanganato. O minoxidil foi usado como titulante na concentração de $2,00 \times 10^{-2} \mathrm{~mol} \mathrm{~L}^{-1}$ diluído em solução de $\mathrm{H}_{2} \mathrm{SO}_{4} 2,00 \mathrm{~mol} \mathrm{~L}^{-1}$. As recuperações para método proposto foram da ordem de 94,5 a 95,2 \% dependendo da amostra comercial. O método proposto foi aplicado à amostras comerciais contendo minoxidil e comparado com resultados obtidos a partir de procedimentos cromatográficos com erros relativos da ordem de -1 a $-5,26 \%$. O procedimento se mostrou simples, rápido e pode ser uma alternativa para a determinação de minoxidil em amostras farmacêuticas, nas quais a concentração do fármaco é relativamente elevada.
\end{abstract}

Palavras Chave: Determinação de minoxidil; titulação, permanganato de potássio.

\section{Introdução}

O minoxidil, 3-óxido-2,4-diamino-6-pipedinopirimidina (Figura 1) é um sólido cristalino branco, inodoro, insolúvel em água em acetona ou soluções alcalinas, é pouco solúvel em álcoois e solúvel em soluções ácidas [1]. O minoxidil age como vasodilatador periférico, quando ingerido é metabolizado no fígado pela ação da sulfotransferase, transformando-se na molécula ativa hidralazina. Como a hidralazina produz apenas vaso-dilatação arteriolar, não tendo ação em vasos de capacitância, causa ativação simpática reflexa, taquicardia e retenção hidro-salina, portanto o minoxidil deve ser administrado em conjunto com diuréticos e bloqueadores simpáticos para minimizar as reações adversas citadas acima [2-4]. 


\section{Sousa et al. Figura 1}

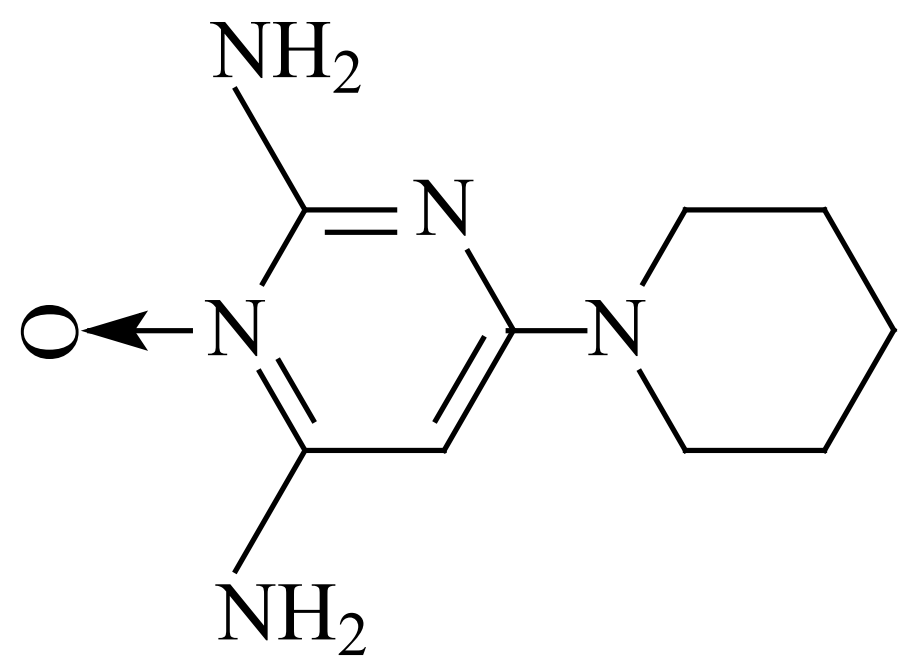

Figura 1: Fórmula estrutural do minoxidil

Devido à sua propriedade vaso dilatadora, o minoxidil é usado no controle da Hipertensão Arterial e no tratamento da Alopécia Areata, um tipo de calvície conhecida vulgarmente como "pelada" [4-8].

A permanganometria se refere às reações redox, nas quais se usa $\mathrm{KMnO}_{4}$ como agente oxidante para a determinação volumétrica de um agente redutor. Este tipo de ração foi proposto inicialmente por F. Margueritte na determinação de $\mathrm{Fe}(\mathrm{II})$ em soluções ácidas. O potencial padrão de redução do $\mathrm{MnO}_{4}^{-}$, em meio de ácido sulfúrico 1 mol L-1 é de 1,52 volts o que indica que o $\mathrm{KMnO}_{4}$ é um oxidante forte (Eq. 1). O meio é acidificado com ácido sulfúrico $\left(\mathrm{H}_{2} \mathrm{SO}_{4}\right)$, pois esse ácido não se oxida frente ao $\mathrm{KMnO}_{4}$, como ocorre com o ácido clorídrico $(\mathrm{HCl})$, que se oxida formando $\mathrm{Cl}_{2}$ reagindo paralelamente [9].

\section{$\mathrm{E}^{\mathrm{o}}=1,52 \mathrm{~V} \quad$ (Eq. 1)}

$\mathrm{MnO}_{4}^{-}+8 \mathrm{H}^{+}+5 \mathrm{e}^{-} \rightarrow \mathrm{Mn}^{2+}+4 \mathrm{H}_{2} \mathrm{O}$

Em meio ácido, o $\mathrm{KMnO}_{4}$, que se apresenta na forma de uma solução de coloração violeta, se reduz a Mn (II), incolor [10,11].

$\mathrm{O} \mathrm{KMnO}_{4}$ apresenta a desvantagem de não ser padrão primário devido à instabilidade à incidência de luz e a instabilidade térmica o permanganato de potássio. Entretanto, apesar disso, o $\mathrm{KMnO}_{4}$ apresenta como vantagens um alto poder oxidante e, ainda, quando usado em titulações em que a substância a ser titulada é incolor ou levemente colorida, o $\mathrm{KMnO}_{4}$ além de oxidante é auto-indicador devido à mudança de coloração de rosa $\left(\mathrm{MnO}_{4}^{-}\right)$para incolor $\left(\mathrm{Mn}^{2+}\right)$. A reação é geralmente lenta à temperatura ambiente, podendo ser acelerada pela temperatura, geralmente na ordem de $60^{\circ} \mathrm{C}[10,11]$.

A análise titrimétrica, ou titulação volumétrica, consiste na determinação exata do volume, de uma solução padrão, necessário para reagir quantitativamente com uma solução cuja concentração será determinada [12]. Trata-se de uma técnica muito utilizada para a determinação quantitativa de diversas substâncias atualmente, mesmo sendo uma técnica clássica de determinação. A Association of Official Analytical Chemists (AOAC) é uma organização internacional com mais de 120 anos de experiência em validar e aprovar métodos para análise de alimentos, medicamentos e produtos agrícolas [13]. A AOAC conta com uma coleção exclusiva de análises químicas. $\mathrm{Na} 18^{\mathrm{a}}$ Edição dos Métodos Oficiais de Análises da AOAC são descritos um total de 2813 métodos analíticos [14], dos quais 442 procedimentos, 16\%, são mé- 
todos titrimétricos [15], mostrando a sua importância e aplicabilidade.

Procedimentos para a determinação de minoxidil tais como eletroquímicos $[16,17]$, espectrofotométricos [18], cromatográfico [19-21] já foram descritos anteriormente.

Com o objetivo de simplificar os procedimentos analíticos e reduzir custos de análise em amostras farmacêuticas com concentrações relativamente elevadas, Sousa e colaboradores [22], propuseram um método de titulação espectrofotométrica baseada na reação redox entre minoxidil em meio ácido e $\mathrm{KMnO}_{4}$. O método monitora o decréscimo da absorbância em $550 \mathrm{~nm}$ peal redução do $\mathrm{KMnO}_{4}$ pelo minoxidil (Eq. 2).

$\mathrm{O}$ presente trabalho tem por objetivo propor uma metodologia simples e de baixo custo para determinação de minoxidil em formulações farmacêuticas de uso tópico. Devido ao grande número de farmácias de manipulação a titulação proposta nesse trabalho pode ser empregada no controle de qualidade dessas farmácias exigindo baixo investimento para a implementação, sem .a necessidade de instrumentação sofisticada.

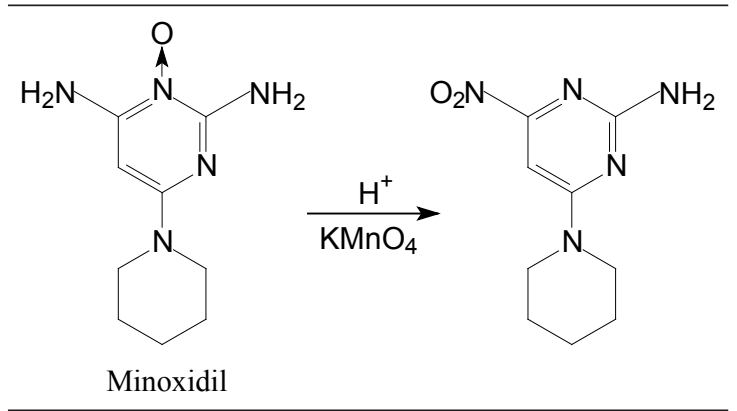

(Eq. 2)

Materiais e Métodos

Reagentes e soluções

Todos os reagentes utilizados no presente trabalho foram grau analítico, PA. As soluções foram preparadas em água bidestilada num destilador de quartzo. As soluções de $\mathrm{KMnO}_{4}$ (Merck) foram preparadas pela dissolução direta do sal em água bidestilada. A solução de $\mathrm{KMnO}_{4}$ foi padronizada com oxalato de sódio (Merck, USA) dissolvido em ácido sulfúrico (Mallinckrodt, México) 2,00 $\mathrm{mol} \mathrm{L}^{-1}$ [23]. O minoxidil foi adquirido da Natural Pharma Produtos Farmacêuticos Ltda, lote $\mathrm{n}^{0} 1901$.

\section{Padronização do permanganato de potássio}

Uma vez que o permanganato não é padrão primário, recomenda-se a padronização de suas soluções com oxalato de sódio em meio ácido. Neste meio o sal do ácido fraco é protonado formando o ácido oxálico. A padronização é realizada adicionando-se o padrão à solução de $\mathrm{KMnO}_{4}$ até 90 a $95 \%$ do volume requerido para o ponto de equivalência calculado previamente a uma temperatura na ordem de $60{ }^{\circ} \mathrm{C}$, tendo como ponto final da titulação uma coloração rosa pálido devido ao pequeno excesso de $\mathrm{KMnO}_{4}$ [23]. Normalmente se utilizam concentrações de $\mathrm{KMnO}_{4}$ da ordem de

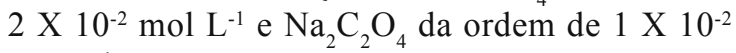
$\mathrm{mol} \mathrm{L}-1$, sob agitação rigorosa.

Titulação de $\mathrm{KMnO}_{4}$ com minoxidil

As titulações foram realizadas adicionando-se solução de minoxidil $\left(0,01 \mathrm{~mol} \mathrm{~L}^{-1}\right)$ em meio de $\mathrm{H}_{2} \mathrm{SO}_{4}\left(2,00 \mathrm{~mol} \mathrm{~L}^{-1}\right)$ à $20,0 \mathrm{~mL}$ de solução de $\mathrm{KMnO}_{4}$ à partir de uma bureta sob a temperatura desejada. O ponto final foi determinado pelo desaparecimento da cor púrpura característica do $\mathrm{KMnO}_{4}$.

\section{Método comparativo}

Foi usado um método comparativo para determinação de minoxidil nas amostras comerciais proposto por Zarghi et al. [21], e que consiste de um procedimento de cromatografia liquida de alta performance (HPLC). Foram encontrados erros relativos de - 3,0\% para o Neoxidil ${ }^{\circledR}$ e 4,0\% para o Regaine ${ }^{\circledR}$ em relação aos valores das quantida- 
des de minoxidil citados nos rótulos, $20 \mathrm{mg} \mathrm{mL}^{-1}$ para ambos.

\section{Resultados e discussão}

Para a otimização da permanganometria para a determinação de minoxidil em amostras farmacêuticas foram estudados o efeito da ordem da adição dos reagentes: qual o melhor titulante e titulado, influência da concentração de $\mathrm{H}_{2} \mathrm{SO}_{4}$, influência das concentrações de minoxidil e permanganato de potássio, influência da temperatura na reação redox. A avaliação do método foi feita com base no estudo da repetibilidade, análise das amostras comerciais e teste de adição e recuperação, conforme se descreve a seguir.

\section{Estudo do efeito da ordem da adição de amostra}

Neste caso investigou-se o efeito da ordem de adição de reagentes, isto é, definiu-se o titulado e o titulante. $\mathrm{Na}$ padronização do $\mathrm{KMnO}_{4}$ este é usado como titulante e auto-indicador, porém na determinação titrimétrica de minoxidil o permanganato, quando usado como titulante auto-indicador impossibilitava a visualização do ponto final da titulação pois, mesmo com um pequeno excesso do permanganato de potássio, a cor rosa pálido, que indicaria o ponto final da titulação, ainda não era observada [23].

Após a adição de excesso de $\mathrm{KMnO}_{4}$ ocorria o aparecimento de um precipitado marrom típico de óxido de manganês IV $\left(\mathrm{MnO}_{2}\right)$, todavia ao inverter a ordem de adição, ou seja, o minoxidil como titulante e o permanganato como titulado, o ponto final da titulação pode ser visualizado no momento da descoloração total da solução de permanganato de potássio. Portanto optou-se pela utilização do minoxidil como titulante e do $\mathrm{KMnO}_{4}$ como titulado. Provavelmente pelo efeito catalítico do $\mathrm{Mn}^{2+}$, na decomposição do $\mathrm{MnO}_{4}^{-}$[8].
Estudo da influência da concentração de ácido sulfúrico

Variou-se a concentração de ácido sulfúrico com o propósito de desenvolver um procedimento envolvendo a menor quantidade de ácido possível, sem deixar de considerar que a redução do $\mathrm{KMnO}_{4}$ em meio ácido requer 8 mols do íon $\mathrm{H}^{+}$ para cada mol de $\mathrm{KMnO}_{4}$ de acordo com a semireação apresentada anteriormente na Equação 1 [9]. O efeito da concentração de ácido sulfúrico na reação redox entre o minoxidil e permanganato de potássio foi investigado no intervalo de 0,100 a 4,00 $\mathrm{mol} \mathrm{L}^{-1}$. Para valores de concentração menores que 2,00 $\mathrm{mol} \mathrm{L}^{-1}$ de ácido sulfúrico formava-se, preferencialmente, $\mathrm{MnO}_{2}$ marcado pela formação de um precipitado marrom indicando que a reação não ocorreu como esperado, com a conversão do $\mathrm{MnO}_{4}^{-}$em $\mathrm{Mn}^{2+}$ [23]. Portanto, a concentração mínima de ácido sulfúrico foi de $2,00 \mathrm{~mol} \mathrm{~L}^{-1}$ para a reação redox entre o minoxidil e permanganato de potássio.

\section{Estudo da influência das concentrações de minoxidil e de permanganato de potássio}

As concentrações de minoxidil e de $\mathrm{KMnO}_{4}$ foram variadas no sentido de se poder trabalhar com amostras mais diluídas quanto possível. Entretanto, concentrações significativamente diferentes de $1,00 \times 10^{-2} \mathrm{~mol} \mathrm{~L}^{-1}$ de minoxidil e $2,00 \times 10^{-2} \mathrm{~mol}$ $\mathrm{L}^{-1}$ de permanganato de potássio, também provocaram a formação de $\mathrm{MnO}_{2}$. Portanto, as concentrações selecionadas se situaram em torno daquelas apresentadas acima tanto para o minoxidil, quanto para o permanganato de potássio. Essas concentrações coincidem com as recomendadas para a padronização do $\mathrm{KMnO}_{4}$ [23], com o oxalato de sódio em meio ácido.

\section{Estudo da influência da temperatura na reação redox}

Por questões relacionadas à cinética de redução do $\mathrm{MnO}_{4}^{-}$, recomenda-se o uso de temperaturas maiores que a ambiente nas reações com este íon. [23], Neste estudo, avaliou-se o tempo decorrido para o consumo do $\mathrm{KMnO}_{4}$ pela adição 
dos $90 \%$ do volume do ponto de equivalência para a solução de minoxidil recomendado para o inicio da titulação. Os resultados são apresentados na Figura 2, que representa a variação do tempo gasto na titulação, em função da temperatura do sistema.

\section{Sousa et al. Figura 2}

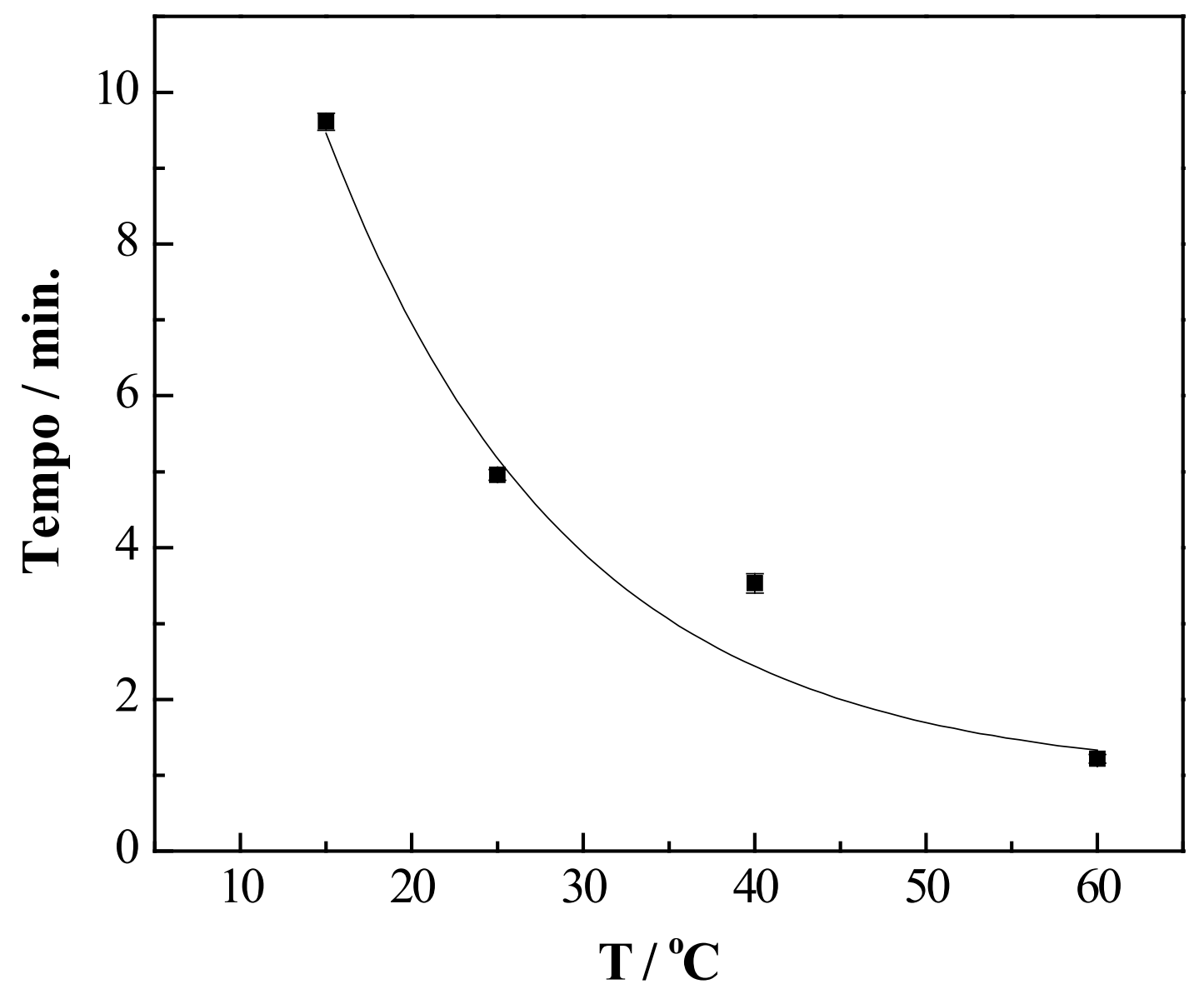

Figura 2: Variação do tempo para consumir 90\% de $\mathrm{MnO}_{4}^{-}$necessário para o ponto final, em função da temperatura do sistema. Cada ponto representa a média, de três determinações.

Volume de $\mathrm{KMnO}_{4}=5,00 \mathrm{~mL}$;

$\left[\mathrm{KMnO}_{4}\right]=2,13 \times 10^{-2} \mathrm{~mol} \mathrm{~L}^{-1} \mathrm{e}$ $[$ minoxidil $]=1,05 \times 10^{-2} \mathrm{~mol} \mathrm{~L}^{-1}$

Observou-se que, em temperaturas maiores do que $60^{\circ} \mathrm{C}$, houve a formação de $\mathrm{MnO}_{2} \mathrm{e}$, portanto, considerou-se esta como a temperatura limite para que não haja a formação do óxido de manganês. Por outro lado, observou-se que titulações realizadas com as mesmas soluções de titulado e titulante, porém em temperaturas menores que $60^{\circ} \mathrm{C}$, apresentaram pontos de equivalência muito próximos entre si, isto é, a temperatura não influencia no ponto final da titulação, mas tem influência no tempo necessário para se consumir $90 \%$ do titulante: quanto maior a temperatura mais rápida a reação, com limite de $60^{\circ} \mathrm{C}$. 
Considerando-se o menor tempo experimental, selecionou-se a temperatura de $60^{\circ} \mathrm{C}$ para os estudos posteriores.

\section{Estudo da repetibilidade}

Avaliou-se a repetibilidade dos resultados titulando-se o padrão de permanganato com uma solução padrão de minoxidil por dez vezes consecutivas. Os resultados são apresentados na Tabela 1 e mostram uma repetibilidade de $6,0 \pm 0,1 \mathrm{~mL}$ para o ponto final, considerando-se os resultados das 10 determinações consecutivas, com erro relativo da ordem de 1\%, considerando-se as concentrações padronizadas dos reagentes.

Tabela 1: Resultados obtidos no estudo da repetibilidade do método titrimétrico com $\mathrm{KMnO}_{4}$

\begin{tabular}{cc}
\hline Replicata & Volume do ponto final $/ \mathbf{m L}$ \\
\hline 1 & 6,10 \\
2 & 6,10 \\
3 & 5,90 \\
4 & 5,95 \\
5 & 6,10 \\
6 & 6,05 \\
7 & 6,05 \\
8 & 5,85 \\
9 & 5,95 \\
10 & 6,15 \\
\hline Média & $6,0 \pm 0,1(\mathrm{n}=10)$ \\
\hline
\end{tabular}

Ponto de equivalência calculado $=5,96 \mathrm{~mL}$;

Erro \% $=[($ média - calculado $) /$ calculado $] .100=1,01 \%$;

Volume de $\mathrm{KMnO}_{4}=5,00 \mathrm{~mL}$;

$\left[\mathrm{KMnO}_{4}\right]=2,13 \times 10^{-2} \mathrm{~mol} \mathrm{~L}^{-1} \mathrm{e}$

$[$ minoxidil $]=1,05 \times 10^{-2} \mathrm{~mol} \mathrm{~L}^{-1}$

\section{Análise das amostras comerciais}

Após a otimização do sistema e o estudo repetibilidade, aplicou-se o método desenvolvido em duas formulações farmacêuticas contendo minoxidil (Neoxidil ${ }^{\circledR}$ e Regaine ${ }^{\circledR}$ ) para uso tópico. Investigou-se a provável interferência do etanol e do propilenoglicol, presentes como veículo nas formulações farmacêuticas [24,25]. Os grupos hidroxila presentes no etanol e no propilenoglicol poderiam ser oxidados pelo permanganato de potássio. Portanto, amostras foram tituladas na presença do veículo e após a extração do mesmo o mesmo, para comparação.
A preparação das amostras das quais não foi extraído o veículo, consistiu na simples diluição de um volume exato da solução tópica em ácido sulfúrico 2,00 $\mathrm{mol} \mathrm{L}^{-1}$, suficiente para atingir a concentração de $1,00 \times 10^{-2} \mathrm{~mol} \mathrm{~L}^{-1}$.

A extração do veículo das formulações farmacêuticas consistiu na retirada do máximo de etanol contido em um volume conhecido de amostra com auxílio de um evaporador rotatório a $70{ }^{\circ} \mathrm{C}$ com pressão reduzida. Nessa etapa, o propilenoglicol (ponto de ebulição $=187,2{ }^{\circ} \mathrm{C}$ ) não é eliminado, devido a seu ponto de ebulição, formou-se uma mistura viscosa de minoxidil e propilenoglicol à qual adicionou-se acetona para 
a precipitação do minoxidil. O minoxidil foi separado por filtração a vácuo, utilizando-se um funil com uma placa sinterizada. Secou-se o precipitado em estufa a aproximadamente $60{ }^{\circ} \mathrm{C}$ por 30 minutos o qual foi diluído em ácido sulfúrico $2,00 \mathrm{~mol} \mathrm{~L}^{-1}$, obtendo-se a solução de trabalho.

Comparando-se os pontos finais das titulações das amostras sem extração ou com extração do veículo, verificou-se que o etanol e propilenoglicol contidos nos fármacos não interferiram no método de determinação proposto para o minoxidil (Tabela 2), já que os resultados concordam entre si em $95 \%$ de confiança de acordo com o teste t-Student [26]. Isto pode ser explicado pela maior facilidade de oxidação do álcool em meio básico, segundo Solomons [27]. No presente procedimento, usando meio ácido, a oxidação do etanol ocorreria com maior lentidão

Tabela 2: Comparação entre os pontos finais das titulações das amostras com e sem extração do veículo

\begin{tabular}{ccc}
\hline Amostras & Com extração do veículo / ml* & Sem extração do veículo / $\mathbf{m L}^{*}$ \\
\hline Neoxidil $^{\circledR}$ & $19.5 \pm 0,03$ & $19,2 \pm 0,12$ \\
Regaine $^{\circledR}$ & $19,6 \pm 0,01$ & $19,8 \pm 0,13$ \\
\hline
\end{tabular}

${ }^{*}$ média \pm desvio padrão, $\mathrm{n}=3$

Portanto decidiu-se analisar a amostras comerciais sem a extração do veículo, devido a maior simplicidade e rapidez do procedimento.

Seguindo os parâmetros definidos nos estudos de otimização do método proposto para a determinação de minoxidil, as amostras comerciais, Neoxidil ${ }^{\circledR}$ e Regaine $^{\circledR}$, foram analisadas e os resultados são apresentados na Tabela 3.

Tabela 3: Resultados obtidos na análise da amostra usando o método proposto, comparados com os valores rotulados e aqueles obtidos com o método comparativo

\begin{tabular}{|c|c|c|c|c|c|}
\hline Amostras & Rótulo a & Titulação ${ }^{\text {b }}$ & Comparativo $^{\text {b }}$ & E1 / \%c & E2 $/ \% \mathrm{~d}$ \\
\hline Neoxidil $^{\circledR}$ & 20,0 & $19,2 \pm 0,12$ & $19,4 \pm 0,41$ & $-4,00$ & $-1,03$ \\
\hline Regain $^{\circledR}$ & 20,0 & $19,8 \pm 0,13$ & $20,9 \pm 0,47$ & $-1,00$ & $-5,26$ \\
\hline
\end{tabular}

$\mathrm{a}$ - valores dados em $\mathrm{mg} \mathrm{mL}^{-1}$

$\mathrm{b}$ - valores dados em $\mathrm{mg} \mathrm{mL} \mathrm{mL}^{-1} \pm$ desvio padrão, $\mathrm{n}=3$

$\mathrm{c}-$ erros relativos ao rótulo

$\mathrm{d}$ - erros relativos ao método comparativo

Os resultados obtidos (Tabela 3) pelo método proposto se mostraram coerentes com os valores rotulados das amostras comerciais e com o método comparativo. Os resultados obtidos pelo método proposto concordam com os valores rotulados das amostras comerciais em 99,9 \% de confiança e concordam em 90,0 \% de confiança com o método comparativo, usando o teste t-Student [26].

\section{Teste de adição e recuperação}

A exatidão do método proposto foi avaliada pelo teste de adição e recuperação, observando-se recuperação entre $94,5 \%$ para o Neoxidil ${ }^{\circledR}, 95,2$ $\%$ Regaine ${ }^{\circledR}$.

\section{Conclusões}

A determinação titrimétrica de minoxidil em formulações farmacêuticas usando $\mathrm{KMnO}_{4}$ mostrou-se um método de aplicação simples e de 
baixo custo. Os valores de concentração de minoxidil obtidos pelo método proposto foram concordantes tanto quanto ao rótulo e tanto quanto ao método comparativo. Portanto, conclui-se que o método aqui proposto é eficiente na determinação de minoxidil em amostras comerciais, dentro das limitações de um procedimento volumétrico clássico.

O método pode ser útil para a determinação e controle de qualidade, em farmácias de manipulação e laboratórios que não disponham de equipamentos mais sofisticados, quando aplicado em amostras com concentrações relativamente elevadas como as formulações farmacêuticas de uso tópico e/ou comprimidos contendo minoxidil.

Determination Of Minoxidil In Pharmaceutical Formulations Using A Permanganometric Titrimetric Procedure

\begin{abstract}
A titrimetric procedure for the determination of minoxidil using $\mathrm{KMnO}_{4}$ as oxidizing agent is described. The best conditions were optimized considering the $\mathrm{H}_{2} \mathrm{SO}_{4}, \mathrm{KMnO}_{4}$ and minoxidil concentrations, the temperature of the system and the order of addition of the reactants. The method was applied to commercial samples and compared with the results from a standard chromatographic procedure. Recoveries from 94.5 to $95.2 \%$ were observed depending on the sample. Comparison with the chromatographic procedure reveled relative errors of -1.00 to $-5.26 \%$. The method was relatively fast, easy to perform and can be a low cost alternative for pharmaceutical samples in which minoxidil concentration is relatively high..
\end{abstract}

KEYWORDS: Minoxidil determination; titration; potassium permanganate.

\section{Referências Bibliográficas}

[1] S. S. Budavari, M.J. O’Neil, A. Smith, P.E. Heckelman, J.F. Kinneary, The Merck Index, $13^{\text {th }}$, Merck \& Company Incorporated, Whitehouse Station, New Jersey, 2001, 6229p.

[2] Medina, A. R.; Córdova, M. L. F.; Molina-Díaz, A., "Integrated flow injection-solid phase spectrophotometric determination of minoxidil". Talanta, 50: 277, 1999

[3] Han, J. H.; Kwon,O. S.; Chung, J. H.; Cho, K. H.; Eun, H. C.; Kim, K. H. "Expression of androgen receptor, estrogen receptor alpha and beta in the dermal papilla of human hair follicles in vivo". J. Dermatol. Science, 36 (3): 176, 2004

[4] Nobre, F.; Lima, N.K.C. "Hipertensão arterial: conceito , classificação e critérios diagnósticos". in manual de cardiologia SOCESP, Atheneu, São Paulo, 2000.

[5] Mano, R. "Manual de hipertensão arterial". Mano, Ladário, 2002

[6] http://www.tuotromedico.com/temas/hipertension.htm, consultado em abril de 2005

[7] Kapln, N.M. Systemis hypertension: mechanism and diagnosis. "In Heart disease" BRANWALD, E.; Zipes, D.P.; Libby, P (eds.). $6^{\text {th }}$. Ed., saunders, Philadelphia, 2001.

[8] Latufo, P.A.; Lolio, C.A. "Epidemiologia da Hipertensão Arterial no Brasil". In SOCESP Cardiologia, Vol. 2, Atheneu, São Paulo, 1996.
[9] Jeffery, G.H.; Bassett, J.; Mendham, J.; Denney, R.C.; Vogel. Analise Química Quantitativa; quinta edição; 260; LTC Ltda.; 1992.

[10] W. Misiuk, H. Puzanowska-Tarasiewics, Anal. Lett., 35 (2002) 1163-1170.

[11] Jeffery, G.H.; Bassett, J.; Mendham, J.; Denney, R.C.; Vogel.Analise Química Qualitativa; quinta edição; 396; LTC Ltda.; 1992.

[12] Jeffery, G.H.; Bassett, J.; Mendham, J.; Denney, R.C.; Vogel.Analise Química Quantitativa; quinta edição; 168; LTC Ltda.; 1992.

[13] Terra, J.; Rossi, A. V. "Sobre o desenvolvimento da análise volumétrica e algumas aplicações atuais". Quim. Nova, 28 (1): 166, 2005

[13] Página da internet acessada no dia 03/08/2008: http:// www.eoma.aoac.org/methods/result.asp?string $=\mathrm{b}$

[15] Página da internet acessada no dia 03/08/2008: http:// www.eoma.aoac.org/methods/result.asp?string $=\mathrm{b}$

[16] Amankwa, L.; Chatten, L.G.; Pons, S.; "Electrochemical studies on minoxidil and determination in tablets by differential-pulse polarography" Analyst, 108: 1221, 1983.

[17] Pfaffen, V.; Ortiz, P.I.; "Electrochemical determination of minoxidil in different Pharmaceutical formulations by flow injection analysis". Analytical Sciences, 22: 91, 2006

[18] Ruiz-Medina, A.; Fernandez-de Cordova, M.L.; MolinaDiaz, A.; "Integrated flow injection-solid phase spectrophotometric determination of minoxidil". Talanta, 50 (2): 277, 1999. 
[19] Zarghi, A.; Shafaati, A.; Foroutan, S.M.; Khoddam, A.; "Rapid determination of minoxidil in human plasma using ion-pair HPLC". J. Pharm. Biomed. Anal, 36: 377, 2004.

[20] Fanali, S.; Cristalli, M.; Catellani, P.; "Determination of minoxidil in pharmaceutical forms by capillary isotachophoresis". J. Chromatogr. A, 405: 385, 1987.

[21] Zarghi, A.; Jenabi, M.; Ebrahimian, A. J. " HPLC determination of the tretonoin-minoxidil solution" Pharm. Acta Helvet., 73: 163, 1998.

[22] Sousa, R. A.; Semaan, F.S.; Fragali, J.A.B.; Cavalheiro E.T.G.; "Fast Determination of Minoxidil by Photometric Flow Titration". Eclética Química, 30 (3): 79, 2005.

[23] Jeffery, G.H.; Bassett, J.; Mendham, J.; Denney, R.C.; Vogel. Análise Química Quantitativa; quinta edição; 268; LTC Ltda. 1992

[24] Karlberg, B.; Pacey, G.E. Flow Injection Analysis: A Practical Guide. Elsevier, Amsterdam, 1989.

[25] Zhong, W. Z. et l, Quantitative analysis of 2-n-nonyl1,3-dioxolane by stable-isotope dilution gas chromatography mass spectrometry. J. Chromatogr. B, 705: 39, 1998.

[26] Harris, D.C. Análise Química Quantitativa, quinta edição, p. 61, LTC - Livros técnicos e Científicos Editora AS, 2001. 